\title{
Current Status and Future Directions for Electronic Point-of-Care Clinical Decision Support to Improve Diabetes Management in Primary Care
}

\author{
Patrick J. O'Connor, MD, MA, MPH, ${ }^{1,2}$ and JoAnn M. Sperl-Hillen, MD ${ }^{1,2}$
}

\begin{abstract}
In the past decade there have been major improvements in the design, use, and effectiveness of point-of-care clinical decision support (CDS) systems to improve quality of care for patients with diabetes and related conditions. Advances in data exchange, data security, and human factors research have driven these improvements. Current diabetes CDS systems have high use rates, high clinician/user satisfaction rates, and have measurably improved glucose control, blood pressure control, and cardiovascular risk trajectories in adults with diabetes. As diabetes care increasingly relies on complex biomarker-driven risk prediction methods to optimize care goals and prioritize treatment options based on potential benefit to an individual patient, CDS systems will become indispensable tools to guide clinician and patient decision-making. In this study we describe specific challenges that must be addressed further to improve the design, implementation, and effectiveness of primary care diabetes CDS systems in coming years.
\end{abstract}

Keywords: Diabetes mellitus, Clinical decision support, Electronic health records, Primary care, Clinical outcomes, Costs of care, Quality of care.

\section{Introduction}

$\mathbf{I}$ N 1991 AND in 1993, the Institute of Medicine issued optimistic reports predicting that implementation of electronic health records (EHRs) would provide the platform needed to deliver point-of-care clinical decision support and usher in a period of rapid improvement in quality of outpatient care, especially for chronic diseases such as diabetes mellitus. ${ }^{1,2}$ However, it was not until around 2005 that EHR use in primary care began to accelerate, and it was not until 2010 that published reports of successful primary care diabetes decision support systems began to appear. The 20-year delay was not due to lack of trying. The literature is replete with reports of pioneering but unsuccessful efforts to improve outpatient diabetes care using clinical decision support (CDS) systems. ${ }^{3-7}$

Diabetes care has attracted many CDS innovators because it is a complex chronic disease that requires clinician and patient attention to at least 10 clinical domains at every visit, including blood pressure (BP), lipid, glucose, tobacco and weight control, aspirin use, evaluation of eye, foot, and renal complications, and key immunizations, including those for influenza, pneumonia, and hepatitis $\mathrm{B} .{ }^{8}$ In reality, there are usually even more things to think about at an office visit, because the majority of adults with diabetes have other chronic conditions such as depression, anxiety, coronary heart disease, congestive heart failure, arthritis, osteoporosis, or various lung conditions such as asthma or emphysema-as well as routine preventive care needs. ${ }^{9,10}$ Complicating matters even more, diabetes care is a team sport, and it is important to keep clinicians, educators, and other care team members on the same page to efficiently use resources and to avoid giving mixed messages to patients.

It is precisely in this type of complicated clinical scenario that CDS algorithms are of potentially the most value. Carefully constructed sets of algorithms can address each of these clinical domains, identify missing information or tests that need updating, and identify which of these many clinical domains may be candidates for changes in treatment that may improve quality of care and/or clinical outcomes. Although CDS algorithms are able to personalize care to the evolving clinical state of each individual patient, they are also able to standardize recommendations, thus reducing undesirable

${ }^{1}$ HealthPartners Institute, Minneapolis, Minnesota.

${ }^{2}$ HealthPartners Center for Chronic Care Innovation, Minneapolis, Minnesota. 
variation in care and assuring that all team members who use the CDS system will consistently be aware of key treatment priorities for each patient.

Diabetes CDS is now evolving rapidly and will become an essential platform to guide personalized diabetes management as the complexity of diabetes management increases and as team models of care become more established. ${ }^{11}$ There are now $>10$ classes of glucose-lowering medications, and substantial variation in benefit even within medication classes. The availability of biomarkers that can guide treatment targets $^{12}$ and choice of maximally beneficial treatments ${ }^{13}$ will require algorithmic assessment of an ever-increasing number of factors that influence clinical outcomes. ${ }^{14}$ The availability of continuous monitoring of glucose and other clinical parameters will require innovation in the communication of risks and benefits to patients, and advances in machine-human interaction technology. The future of diabetes CDS is hard to fully envision, but there is no doubt that this technology will be a cornerstone of future diabetes care in both primary care and subspecialty venues, and will enable new patient-direct applications that will likely revolutionize diabetes care as well as chronic disease care in general. ${ }^{15} \mathrm{We}$ live in exciting times. Let us take a look at where we have been, and where we are going.

\section{Priorities Wizard: A Current Diabetes Clinical Decision Support System Used in Primary Care}

Let us start with a "use case." Examining lessons learned from both successful and failed efforts at diabetes CDSguided design of a current CDS system called "Priorities Wizard." This nonproprietary primary care CDS system was developed starting in 2004 using National Institutes of Health research grant funding, and is currently used by medical groups that care for $>3$ million patients in 10 U.S. states.

Priorities Wizard takes EMR data and securely sends it to a Web service where CDS algorithms based on current national evidence-based clinical guidelines parse data for every adult patient visit to answer three questions about that patient: (1) Is this patient at, or not at, recommended personalized treatment goals for diabetes or certain other related chronic conditions? (2) Based on current treatment, treatment goals, allergies, comorbidities, and renal, hepatic, and cardiac status, what evidence-based treatment recommendations may, if implemented, be of potential benefit to this patient at this point in time? (3) Which treatment recommendations should be prioritized based on magnitude of potential benefit to the patient?

Figures 1 and 2 shows two displays of the CDS output from Priorities Wizard. Figure 1 is a one-sided black-and-white printed page given by the rooming staff to a targeted patient after vital signs have been entered into the EMR but before the clinician enters the clinic examination room. Figure 2 is a one-sided black-and-white printed page that the rooming staff put on the examination room door for the clinician to review just before entering the room.

We will interrogate this CDS system to identify its strengths and key opportunities to improve it moving forward. However, it is worth noting that in a series of randomized trials, Priorities Wizard has been shown to improve BP control, glucose control, smoking cessation, and cardiovascular risk, reduce clinical inertia, and improve recognition and diagnosis of diabetes in those who meet clinical criteria for the disease. ${ }^{16-20}$ Moreover, a formal cost-effectiveness analysis shows a base-case estimate of $\$ 3017$ per quality adjusted life year gained, and the CDS system is likely to be cost saving to payers at scale. ${ }^{21}$

\section{Should Diabetes CDS Stand Alone, or Be Integrated with CDS for Other Conditions?}

In the primary care setting, clinicians typically address four to five distinct clinical domains per visit, and many patients, especially those with diabetes, are likely to have multiple other chronic conditions. ${ }^{9,10,22}$ In this work environment it is unreasonable to expect clinicians to consult more than one CDS system at a given clinical encounter. Moreover, primary care clinicians are trained to deliver care that is patient centered, to elicit and address the patient's reason for each visit, and to develop or negotiate the care plan with the patient. ${ }^{23,24}$

It is a challenge to create a CDS system that encompasses multiple disease domains, but in primary care this is an essential cornerstone of CDS systems that will actually be used by primary care clinicians. In the case report earlier, the CDS started with only glucose, BP, and lipid managing suggestions, but now has been expanded to include multiple other care domains such as medication adherence, retinopathy screening, nephropathy screening, weight management, tobacco management, aspirin use, chronic kidney disease management, identification and management of prediabetes, serious mental illness, cancer prevention services, opioid use disorder, and others. The goal of primary care CDS is to include all common chronic and preventive care domains whose care can be reviewed and advanced by the use of algorithms. The breadth of clinical domains included will expand as device-generated data (such as continuous glucose monitoring data) and patient reported data (such as hypoglycemia symptoms and physical activity data) are more systematically collected and merged with existing EHRderived data resources.

This patient-centered rather than disease-centered approach to CDS is essential in primary care. In subspecialty care settings it may also be useful. ${ }^{25}$ For example, in Minnesota endocrinology practices, rates of $\mathrm{BP}$, and lipid control were worse in patients receiving care from endocrinologists than from primary care clinicians. ${ }^{26}$ Part of this is attributable to the complexity of the endocrinology patient population. However, it appears that at least part of the deficits in BP and lipid management in these patients may be due to a narrow view of diabetes care as primarily concerned with glucose control, while not giving adequate attention to other key clinical domains that substantively affect the diabetes patient's risk of a myocardial infarction or stroke. ${ }^{11}$

\section{Should Evidence-Based Care Recommendations Be Prioritized, and if so, How?}

Even with a narrow focus on the $10+$ key elements of diabetes care, prioritization of treatment recommendations is an important feature of effective CDS. The importance of prioritizing clinical care recommendations increases exponentially as the breadth of CDS increases. A classic publication in 2002, enumerated $>600$ key evidence-based elements of care for adults, and showed using chart review 


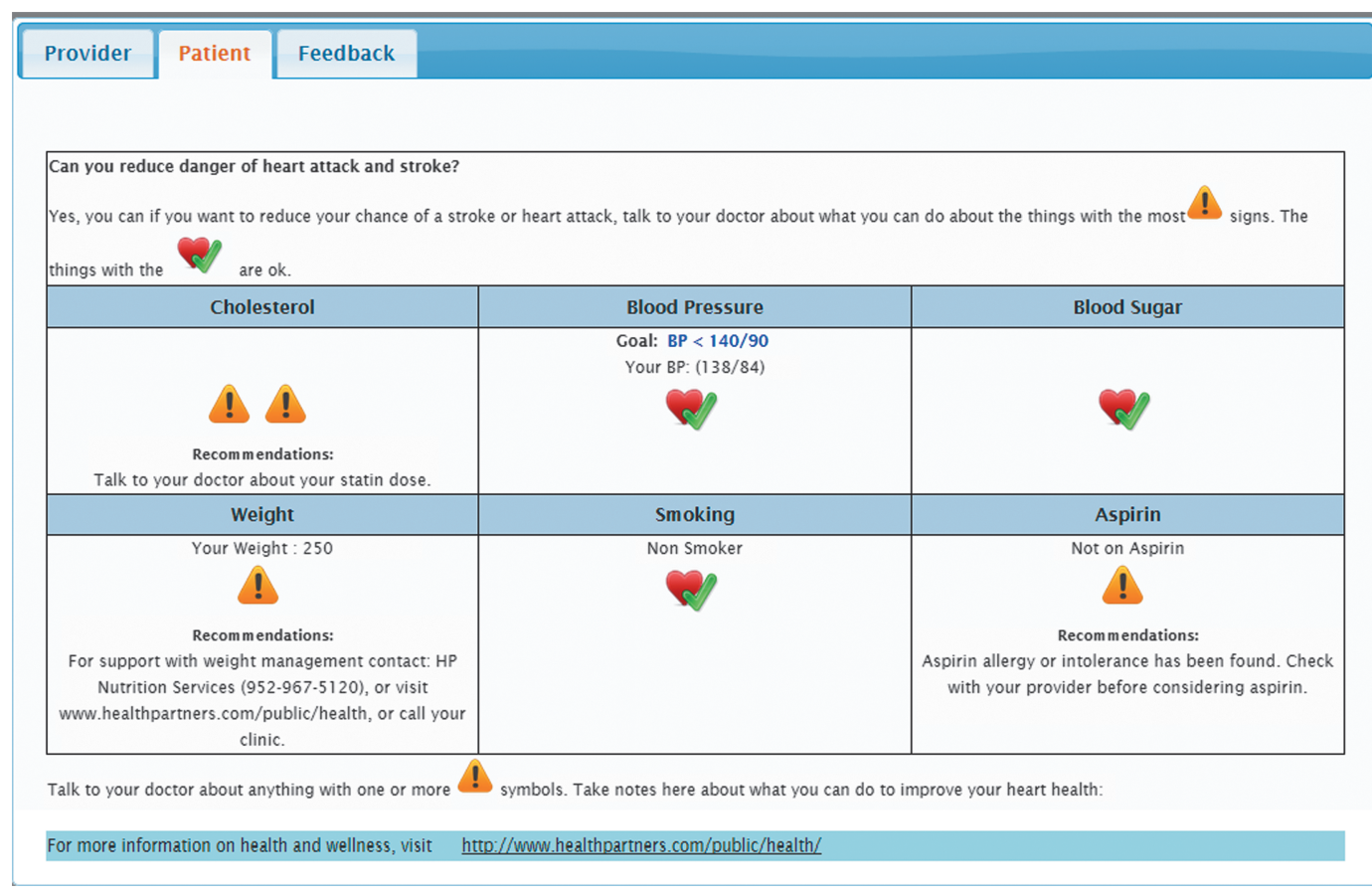

FIG. 1. Patient view of clinical decision support output focused on diabetes and cardiovascular risk factor control. BP, blood pressure.

that only about half of these evidence-based care recommendations were delivered to patients. ${ }^{27}$ They noted that many patients have not only one or two, but dozens of care elements that may be of some benefit.

However, not all those evidence-based elements of care are of equal benefit to a given patient at a given point in time. When one west coast care delivery system enumerated these dozens of "care deficits" and presented them on the opening EHR screen at primary care encounters, most primary care clinicians (PCCs) found the information to be useless because the lists were so long, the visits so short, and the list did not designate which among dozens of care recommendations should be prioritized based on potential benefit to a given patient.

For diabetes patients, it is possible to prioritize care recommendations that are primarily intended to reduce cardiometabolic complications by using risk prediction equations such as the United Kingdom Prospective Diabetes Study (UKPDS) Outcomes Model 2, or the American College of Cardiology/American Heart Association (ACC/AHA) 10year cardiovascular disease (CVD) risk equation for risk of fatal or nonfatal heart attack or stroke. ${ }^{28,29}$ Figures 1 and 2 show patient and clinician CDS output that prioritizes across six key clinical domains (BP, glucose, lipid, weight, tobacco management, and aspirin use) related to major diabetes complications. The risk prediction equations are "behind the screen," but the PCC and patient can see the priority of actions based on potential benefit, and the PCC is given numerical estimated of 10-year CVD risk, and the absolute risk reduction that would be anticipated from recommended actions for suboptimally managed clinical domains.

In "future state" primary care CDS systems, prioritization of treatment options based on potential benefits (and risks) to patients will be challenging because the "unit of benefit" varies across clinical domains. For example, what is the relative benefit of depression treatment, compared with improved glucose or BP control? Innovative methods to accurately prioritize clinical options across multiple clinical domains are very much needed.

\section{How Can CDS Support More Effective Team-Based Diabetes Care?}

CDS systems may enhance diabetes care by serving as a coordinating point for care delivered by numerous diabetes care team members who communicate with one patient. As can be seen in Figures 1 and 2, the CDS output identified high clinical priorities for a given patients, and the recommendations change over time as the patient state evolves. This is also potentially a very good way to identify patients who may benefit from additional attention from pharmacists, case managers, or diabetes educators. The CDS provides a de facto care plan that keeps track of issues that, if addressed, are likely to have the most benefit to an individual patient.

\section{Should CDS Output Be Presented to Patients as Well as to Clinicians?}

Reviews of the effectiveness of diabetes and other chronic disease CDS systems indicate that presenting the CDS output to both the patient and the PCC increases the likelihood that the CDS will have a significant positive impact on intermediate care outcomes such as BP and glucose control.

The CDS display for patients shown in Figure 1 supports shared decision-making (SDM). The rooming staff print the sheet and hand it to the patient before the PCC entering the examination room, saying "You may be able to reduce your danger of a stroke or heart attack if you addressed the things marked with the caution sign. If you are interested in any of 


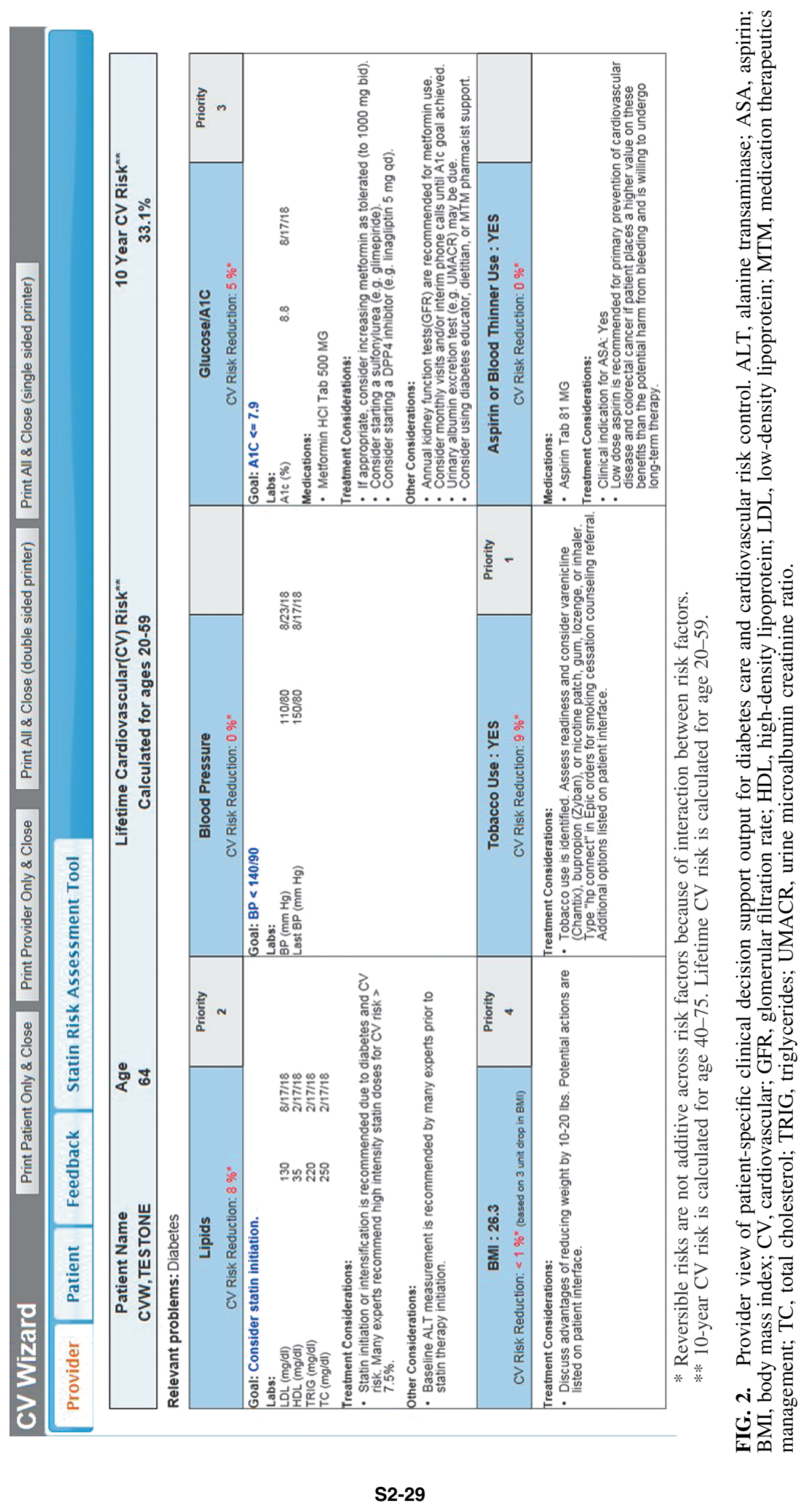


those things, you may want to mention it today to your doctor."

Study done by Greenfield et al. in the 1980s showed that coaching diabetes patients right before a visit improved glucose control, quality of life, and patient satisfaction with care. ${ }^{30}$ However, face-to-face previsit coaching is impractical in most primary care clinic settings. Figure 1 aims to provide a bit of previsit coaching to the patient in an efficient sustainable way. It may serve to direct the patient's attention to actions that are likely to be beneficial and away from actions that do not have much benefit.

\section{What Is the Optimal Way to Provide Risk and Benefit Information to Patients and Clinicians?}

There is considerable uncertainty about how to format and present CDS output in a way that is clear and understandable for patients. ${ }^{31}$ This challenge is accentuated by variation in numeracy and health literacy of patients, and by a lack of consistent results in studies of the effectiveness of various ways to communicate benefits and risks of various treatment options to patients. ${ }^{32,33}$ Montori and colleagues at Mayo Clinic have developed a promising alternative ways to express risks and benefits to patients. ${ }^{34,35}$ However, the impact of risk presentation methods on patient decision-making is not well understood. Ideally, the format of CDS output, including representations of risk and benefit, would be further customized to the health literacy and numeracy of each patient. The ethics of presenting balanced and unbiased representations of benefits and risks is another important area of inquiry.

As shown in Figure 2 clinician CDS output is displayed in a single format, but CDS content is personalized to the current clinical state of each patient. Changes in risk that may be obtained from specific clinical actions are based on standardized risk prediction equations such as the ACC/AHA 10year cardiovascular disease equations, ${ }^{29}$ and published UKPDS risk prediction models. ${ }^{28}$ Preferred drugs vary across medical groups, and care recommendations mention the preferred drug within a class, for example, "Consider initiating an ACE inhibitor, such as lisinopril.’

\section{How Can SDM Be Done in a Time-Efficient Manner?}

Recently some insurers (such as the U.S. Centers for Medicare and Medicaid Services) have required use of certain allegedly standardized and validated SDM tools as a condition for paying for high-tech imaging studies such as lung cancer screening. Several proposed SDM tools are encyclopedic in scope, some as long as eight single-spaced pages in length. In an NIH-funded study, we deployed multipage standardized SDM tools for colorectal and lung cancer screening. These tools are three to eight pages long, were rarely printed or reviewed, and were nearly universally condemned and ignored by PCCs in randomized intervention clinics. We concluded that deploying encyclopedic SDM tools at brief primary care encounters will be futile. Unwieldy and inefficient SDM tools consume precious time and resources, and their enforced use might erode the quality of overall primary care, and increase clinician dissatisfaction and burnout.

Perhaps more flexible and time-efficient SDM approaches will prevail. As noted, the CDS display for patients shown in
Figure 1 is a flexible and very efficient SDM tool. After the patient has reviewed the page they are handed, the PCC can simply ask "Are you interested in addressing any of the things marked with caution signs?" If the patient is not interested, a "precontemplator" message of the type developed by Prochaska et al. is given and discussion moves on to another topic, with a chance to revisit these issues at the next visit. $^{36}$ If the patient is interested, the PCC has saved a great deal of time by not needing to "run the list" of options one by one. Moreover, once the patient has proactively indicated interest, the patient may be more likely to follow through on a mutually agreed upon treatment plan to address their BP, lipids, glucose, smoking, or other clinical priorities. Deploying efficient SDM strategies of various types by link to a Web service before a visit may lead to higher use rates.

\section{What Strategies Can Be Used to Achieve and Maintain High CDS Use Rates?}

Examination of failed pioneering efforts to provide chronic disease and diabetes CDS revealed that most of the failures were related to PCC nonuse of the CDS system or tool. Low CDS use rates were related to multiple factors, such as presenting the CDS too late in the visit (ideally CDS would be seen just before the visit, or near the beginning of the visit), requiring too many clicks to mobilize or use the $\mathrm{CDS}$, relying on clinicians rather than rooming staff to trigger display of the CDS, or CDS output that was too complex to quickly assimilate and act on.

Another reason for nonuse was the "black-box problem," which reflects clinician skepticism of CDS that is generated by complex differential equations or artificial intelligence (AI) methods that are proprietary and/or invisible or beyond the comprehension of the clinician or patient. ${ }^{37}$ It is possible that a variation of this "black-box problem" will re-emerge soon as AI and machine learning approaches are more widely used by CDS developers.

Achieving and maintaining high CDS use rates is a key first step required for CDS success, because CDS that is not used is unlikely to be successful. We have considered four distinct strategies to achieve and maintain high CDS use rates in primary care settings. First, we do not target all patient visits for CDS. We limit CDS deployment to patients who have a potential benefit related to a change in treatment. For example, a diabetes patient who is up to date and under control on the 10 aspects of diabetes care listed earlier would not be targeted for automatic deployment of CDS. In contrast, if a PCC wants to see the CDS for such a patient, it can be triggered manually.

Second, adequate training of staff and clinicians in CDS use is important. However, training can be done in many ways, ranging from face-to-face sessions over lunch in the clinic, to Webinars over lunch, to online training without lunch done asynchronously using local training methods, or other methods. It is notable that to date, the ideal time interval to do retraining is unknown. When there are major updates to clinical guidelines or CDS interfaces, retraining using one of these listed approaches needs to be done.

Third, in our experience across multiple primary care clinic networks, providing PCC- and clinic-specific monthly feedback on CDS use rates at targeted visits is a key to achieving and especially to maintaining high CDS use rates. 
We name the PCCs and the clinics to take advantage of the natural competitive instincts that prevail among clinicians and clinics, who all want to improve the quality of their care. We have worked with one delivery system that did not want feedback; in that system training led to high CDS use rates, but these gradually deteriorated in 2 years due to lack of feedback and lack of advocacy by clinical leaders for regular CDS use.

Fourth and finally, in our early CDS studies we provided financial compensation for nurses and clinics that achieved CDS use rates of $70 \%$ or more of targeted visits. We subsequently discovered that these incentives were not needed to maintain high use rates-systems with and without these incentives achieved similar CDS use rates, using the CDS at $70 \%-80 \%$ of targeted primary care visits. However, use rates appear to deteriorate slightly as the proportion of visits that are targeted for CDS increases. In our experience to date, high CDS use rates can be achieved when targeting up to $30 \%$ of all adult care visits for CDS use. ${ }^{17}$

\section{What Are the Costs of Developing, Implementing,} and Maintaining Outpatient Diabetes CDS Systems?

It would seem logical for EMR vendors to develop and maintain sophisticated CDS systems, but to date, this has happened only as required by law for high-tech imaging procedures, as well as display of rudimentary and very general prompts and reminders (such as "this patient has diabetes and A1c is over goal," or "this patient is due for a pneumonia immunization or breast cancer screening procedure").

There are several reasons why EMR vendors have been slow to develop or host more sophisticated CDS systems. One is the cost of developing and maintaining many clinical algorithms, which may require purchase of guidelines at substantial cost from guideline writing organizations. An additional concern is that not all PCCs will agree with all clinical guideline recommendations, as illustrated recently by extreme controversy about changes in the hypertension treatment guidelines issued in 2017. ${ }^{38,39}$ A third concern in the EMR vendor community is potential medicolegal liability if CDS recommendations are erroneous, or implemented in a suboptimal way by clinicians.

The translation of a written guideline to algorithms, programmed code, and then go-live in clinics is a complicated process. ${ }^{40,41}$ It takes a great deal of clinician and programmer time to move from a written guideline to a reliable EHRlinked Web-based CDS system that operates in real time at multiple clinical venues. Our team has thus far integrated CDS for about 17 conditions, in the Priorities Wizard CDS system described earlier.

A major obstacle to broad implementation of effective CDS systems is the current status of interoperability standards, including HL7 and FHIR data systems, which are not developed enough to support advanced real-time CDS at the time of a clinical encounter.

\section{Can CDS Increase Medical Group Revenue?}

Formal cost-effectiveness evaluations of CDS systems are rare. In a formal analysis done from the perspective of the payer, Gilmer et al. found that a diabetes CDS system was cost-effective, and would likely be cost saving to payers when applied to a larger group of patients than the 20 clinics involved in his formal study. ${ }^{21}$
However, few data are available to establish the business case for CDS use from the point of view of a medical group or care delivery system. Recent analyses have shown that chronic disease CDS systems may increase recognition and coding of diagnoses (such as diabetes) when CDS focuses attention on that condition. Moreover, CDS use also may lead to increase billing, with slightly higher revenue to the medical group per encounter when CDS was used, presumably related to provision of more complex chronic disease care at those encounters. Current studies are focusing on whether including medication adherence data within the CDS displays for the clinician (Fig. 2) will further improve BP, lipid, or glucose control, or have a measurable impact on clinical inertia (NCT01420016, NCT03890588).

\section{What Is the Role of CDS in the Evolving Learning Health Care System?}

Data that are retained in the EHR can be analyzed to assess quality of care, and to assess variation in quality of care across groups of patients, clinics, and individual clinicians. When EHR data are processed through Web-based CDS algorithms, the usual EHR data are greatly enhanced and as many as several hundred specific clinical recommendations that are potentially indicated at a clinical encounter are identified by sophisticated CDS algorithms, and could be stored in residual databases within the care delivery system with appropriate authorizations and data security arrangements.

Careful analysis of CDS-enriched clinical data can indicate specific areas of strength or specific care improvement opportunities at the individual PCC level. These data can guide development of PCC-specific learning or care improvement interventions that have the potential to further improve quality of care in specific clinical circumstances.

As an example, consider two PCCs both of whom have $50 \%$ of their eligible diabetes patients at the composite goal of simultaneously achieving glycated hemoglobin (A1c) $<8 \%, \mathrm{BP}<140 / 90 \mathrm{mmHg}$, on a statin, current nonsmoker, and on aspirin if the patient also had coronary heart disease. Although both PCCs have identical quality scores, the path to better diabetes care for the first PCC involves more frequent use of diuretics in patients with uncontrolled hypertension already on other BP-lowering medications, better recognition and treatment of depression in patients with uncontrolled A1c, and more frequent use of medications to support smoking cessation. In contrast, the path to better diabetes care for the second PCC involves earlier initiation of basal insulin in patients with uncontrolled A1c, recognition of nonadherence to BP medications, and increasing statin doses in diabetes patients who may benefit from moderate to high statin doses, rather than low statin doses.

The potential use of CDS-enhanced EHR data to improve care is exciting, but depends on development of effective interventions that can be tailored to PCC-specific care improvement opportunities. There is considerable data to suggest that simple feedback of information may have a beneficial effect on patterns of care. PCC-tailored simulated case-based learning interventions that match case selection to care improvement opportunities and include learning feedback has also been shown in multiple randomized trials to improve quality of diabetes care by PCCs and primary care 
residents. ${ }^{42,43}$ Other interventions are currently under development by many research groups. ${ }^{44-47}$

\section{Can Diabetes CDS Be Electronically Delivered Directly to Patients Without Clinicians or Clinics?}

The U.S. Food and Drug Administration (FDA) has the responsibility for assuring that patient-direct CDS systems, which can be delivered directly to patients outside traditional clinical encounters, are safe and evidence based. It is clear that there are various electronic pathways that are accessible to many diabetes patients, and that CDS could be delivered from Web services directly to patients through these routes, if the patient first inputs certain health-related data to the Web service. Although many ethical, regulatory, and operational challenges to these evolving technologies exist, it is clear that this is an exciting research frontier that has substantial potential to contribute to changes in diabetes care that may often be beneficial to patients.

\section{How Can Patient-Reported and Device-Derived Data Be Efficiently Incorporated into CDS Algorithms?}

Capturing and feeding patient-reported data into CDS algorithms has great potential to refine and optimize care recommendations and personalize them to specific concerns of a patient. Important patient-reported information that could be considered for inclusion in algorithmic CDS might include things such as recent hypoglycemic symptoms, improvement or deterioration in symptoms potentially related to depression or anxiety, or having higher readiness to change for smoking than what was previously noted.

Including device-derived data, especially data from frequent self-monitored glucose or from continuous glucose monitoring (CGM), could lead to substantial improvement in glucose management especially for patients with frequent mild or serious hypoglycemic events. Hypoglycemia symptoms may prompt modification of insulin or other medication doses, or a revision in A1c goals, or other actions to reduce the likelihood of recurrent or serious hypoglycemia. However, if patients or their devices report hypoglycemic episodes in real time, the care delivery system must respond promptly to such information. The potential difficulty of locating and communicating with a patient who is cognitively impaired by very low sugar is obvious.

\section{Can CDS Systems Efficiently Identify Patients Eligible for Specific Research Studies?}

CDS systems that operate in primary care or other settings are well positioned to identify and begin recruitment of patients who meet certain eligibility criteria for various research studies. The CDS-informed PCC is well positioned to consider informing a patient of the potential availability of a relevant research study, and to take next steps if a patient interested in exploring that option in more detail. We have used Priorities Wizard for this purpose in a large ongoing randomized trial of pharmacist-supported $\mathrm{BP}$ control in adults with hypertension.

\section{Are Current Primary Care Diabetes CDS Systems Relevant for Specialty Care?}

Although we have focused this discussion on applications of diabetes CDS systems in primary care settings, there is reason to believe that such CDS systems may also have some appeal to diabetes educators, diabetes nurse clinicians, and endocrinologists. ${ }^{11,25}$ In our large medical group, Priorities Wizard has been implemented at cardiology and endocrinology clinics, by diabetes educators, and by case managers, at their request.

\section{Summary}

CDS used in primary care and other settings to improve care of patients with diabetes and related conditions has improved dramatically in the past decade. Advances in health informatics, computational efficiency, human factors science, and decision science have led to improved quality of care and high rates of clinician and patient satisfaction with diabetes CDS systems in primary care settings. However, achieving the desired ideal future state of a primary care-oriented CDS system will require more development in interoperability, data security, interface design, and communication of benefits and risks of treatment options to patients and clinicians. Establishing a business case for CDS for care delivery systems under various risk-sharing arrangements remains an unmet need. However, as diabetes care increases in complexity and relies more on biomarker-driven risk prediction science, CDS systems will evolve and likely play an indispensable role by identifying, prioritizing, and presenting algorithm-derived optimal care recommendations to both clinicians and patients.

\section{Author Disclosure Statement}

No competing financial interests exist.

\section{References}

1. Institute of Medicine: The Computer-Based Patient Record. An Essential Technology for Health Care. Washington, DC: National Academy Press, 1991.

2. Institute of Medicine: Guidelines for Clinical Practice. From Development to Use. Washington, DC: National Academy Press, 1992.

3. Montori VM, Dinneen SF, Gorman CA, et al.: The impact of planned care and a diabetes electronic management system on community-based diabetes care: the Mayo Health System Diabetes Translation Project. Diabetes Care 2002;25:1952-1957.

4. Simon SR, Trinacty CM, Soumerai SB, et al.: Improving diabetes care among patients overdue for recommended testing: a randomized controlled trial of automated telephone outreach. Diabetes Care 2010;33:1452-1453.

5. Bright TJ, Wong A, Dhurjati R, et al.: Effect of clinical decision-support systems: a systematic review. Ann Intern Med 2012;157:29-43.

6. Roshanov PS, Fernandes N, Wilczynski JM, et al.: Features of effective computerised clinical decision support systems: meta-regression of 162 randomised trials. BMJ 2013;346:f657.

7. Roshanov PS, Misra S, Gerstein HC, et al.: Computerized clinical decision support systems for chronic disease management: a decision-maker-researcher partnership systematic review. Implement Sci 2011;6:92.

8. American Diabetes Association: Standards of medical care in diabetes 2019. Diabetes Care 2019;42(Suppl 1):S1-S193.

9. Yawn B, Goodwin MA, Zyzanski SJ, Stange KC: Time use during acute and chronic illness visits to a family physician. Fam Pract 2003;20:474-477. 
10. Crabtree B, Miller W, Aita V, et al.: Primary care practice organization and preventive services delivery: a qualitative analysis. J Fam Pract 1998;46:403-409.

11. Vigersky RA, Fitzner K, Levinson J, Diabetes Working G: Barriers and potential solutions to providing optimal guideline-driven care to patients with diabetes in the U.S. Diabetes Care 2013;36:3843-3849.

12. Shah HS, Gao H, Morieri ML, et al.: Genetic predictors of cardiovascular mortality during intensive glycemic control in type 2 diabetes: findings from the ACCORD clinical trial. Diabetes Care 2016;39:1915-1924.

13. Ismail-Beigi F, Moghissi E, Tiktin M, et al.: Individualizing glycemic targets in type 2 diabetes mellitus: implications of recent clinical trials. Ann Intern Med 2011;154: 554-559.

14. Simon GE, Johnson E, Lawrence JM, et al.: Predicting suicide attempts and suicide deaths following outpatient visits using electronic health records. Am J Psychiatry 2018;175:951-960.

15. Koopman RJ, Kochendorfer KM, Moore JL, et al.: A diabetes dashboard and physician efficiency and accuracy in accessing data needed for high-quality diabetes care. Ann Fam Med 2011;9:398-405.

16. O'Connor PJ, Sperl-Hillen JM, Rush WA, et al.: Impact of electronic health record clinical decision support on diabetes care: a randomized trial. Ann Fam Med 2011;9:1221.

17. Sperl-Hillen JM, Crain AL, Margolis KL, et al.: Clinical decision support directed to primary care patients and providers reduces cardiovascular risk: a randomized trial. J Am Med Inform Assoc 2018;25:1137-1146.

18. Rindal DB, Rush WA, Schleyer TK, et al.: Computerassisted guidance for dental office tobacco-cessation counseling: a randomized controlled trial. Am J Prev Med 2013;44:260-264.

19. Kharbanda EO, Asche SE, Sinaiko AR, et al.: Clinical decision support for recognition and management of hypertension: a randomized trial. Pediatrics 2018;141.

20. Sperl-Hillen JM, Rossom RC, Kharbanda EO, et al.: Priorities Wizard: Multisite Web-based primary care clinical decision support improved chronic care outcomes with high use rates and high clinician satisfaction rates. EGEMS (Wash DC) 2019;7:9.

21. Gilmer TP, O'Connor PJ, Sperl-Hillen JM, et al.: Costeffectiveness of an electronic medical record based clinical decision support system. Health Serv Res 2012;47:21372158.

22. Schmittdiel JA, Raebel MA, Dyer W, et al.: Prescription medication burden in patients with newly diagnosed diabetes: a SUrveillance, PREvention, and ManagEment of Diabetes Mellitus (SUPREME-DM) study. J Am Pharm Assoc (2003) 2014;54:e251-e259.

23. Starfield B: Primary Care: Concept, Evaluation and Policy. New York: Oxford University Press, 1992.

24. Lee TH, Bodenheimer T, Goroll AH, et al.: Perspective roundtable: redesigning primary care. N Engl J Med 2008; 359:e24.

25. Rodbard D, Vigersky RA: Design of a decision support system to help clinicians manage glycemia in patients with type 2 diabetes mellitus. J Diabetes Sci Technol 2011;5: 402-411.

26. Minnesota Community Measurement: Minnesota Health Scores Clinic Ratings. 2019. www.mnhealthscores.org/search/ site/Endocrine/bundle/clinic/topics/28/\#/results?topics=M28\& viewmode $=$ detail $\&$ page $=7 \&$ non_rpt_hidden $=y \&$ column name $=\mathrm{M} 28 \&$ columntosort=M28\&sortorder $=$ desc (accessed April 19, 2019).

27. McGlynn EA, Asch SM, Adams J, et al.: The quality of health care delivered to adults in the United States. N Engl J Med 2003;348:2635-2645.

28. Hayes AJ, Leal J, Gray AM, et al.: UKPDS outcomes model 2: a new version of a model to simulate lifetime health outcomes of patients with type 2 diabetes mellitus using data from the 30 year United Kingdom Prospective Diabetes Study: UKPDS 82. Diabetologia 2013;56:1925-1933.

29. Goff DC, Jr., Lloyd-Jones DM, Bennett G, et al.: 2013 ACC/AHA Guideline on the assessment of cardiovascular risk: a Report of the American College of Cardiology/ American Heart Association Task Force on Practice Guidelines. Circulation 2013;129(25 Suppl 2):S49-S73.

30. Greenfield S, Kaplan SH, Ware JE, Jr., et al.: Patients' participation in medical care: effects on blood sugar control and quality of life in diabetes. J Gen Intern Med 1988;3: 448-457.

31. Navar AM, Wang TY, Mi X, et al.: Influence of cardiovascular risk communication tools and presentation formats on patient perceptions and preferences. JAMA Cardiol 2018 [Epub ahead of print]; DOI: 10.1001/jamacardio .2018.3680.

32. Eaton CB: Using cardiovascular age equivalent to close the treatment gap for dyslipidemia. Arch Intern Med 2007;167: 2288.

33. Eaton CB, Parker DR, Craft J, et al.: Using e-health to improve cholesterol management in primary care practice. J Med Pract Manage 2009;24:224-230.

34. Mullan RJ, Montori VM, Shah ND, et al.: The diabetes mellitus medication choice decision aid: a randomized trial. Arch Intern Med 2009;169:1560-1568.

35. Mann DM, Ponieman D, Montori VM, et al.: The Statin Choice decision aid in primary care: a randomized trial. Patient Educ Couns 2010;80:138-140.

36. Prochaska JO, Redding CA, Evers KK: The Transtheoretical Model and Stages of Change. In: Glanz K, Lewis FM, Rimer BK, eds. In Health Behavior and Health Education, 2nd ed. San Francisco: Jossey-Bass, 1997:60-84.

37. Eddy DM, Schlessinger L: Validation of the archimedes diabetes model. Diabetes Care 2003;26:3102-3110.

38. Whelton PK, Carey RM, Aronow WS, et al.: 2017 ACC/ AHA/AAPA/ABC/ACPM/AGS/APhA/ASH/ASPC/NMA/ PCNA Guideline for the prevention, detection, evaluation, and management of high blood pressure in adults: a report of the American College of Cardiology/American Heart Association Task Force on Clinical Practice Guidelines. June 1, 2018; https://doi.org/10.1161/HYP .0000000000000065 Hypertension 2018;71:e13-e115.

39. Grundy SM, Stone NJ, Bailey AL, et al.: 2018 AHA/ACC/ AACVPR/AAPA/ABC/ACPM/ADA/AGS/APhA/ASPC/ NLA/PCNA guideline on the management of blood cholesterol. Circulation 2018:CIR0000000000000625.

40. Bates DW, Kuperman GJ, Wang S, et al.: Ten commandments for effective clinical decision support: making the practice of evidence-based medicine a reality. J Am Med Inform Assoc 2003;10:523-530.

41. O'Connor PJ, Sperl-Hillen JM, Fazio CJ, et al.: Outpatient diabetes clinical decision support: current status and future directions. Diabet Med 2016;33:734-741.

42. O'Connor PJ, Sperl-Hillen JM, Johnson PE, et al.: Simulated physician learning intervention to improve safety and 
quality of diabetes care: a randomized trial. Diabetes Care 2009;32:585-590.

43. Sperl-Hillen JM, O'Connor PJ, Rush WA, et al.: Simulated physician learning program improves glucose control in adults with diabetes. Diabetes Care 2010;33:1727-1733.

44. Sperl-Hillen J, O'Connor P, Ekstrom H, et al.: Using simulation technology to teach diabetes care management skills to resident physicians. J Diabetes Sci Technol 2013;7: 1243-1254.

45. Zurawski A, Komaromy M, Ceballos V, et al.: Project ECHO brings innovation to community health worker training and support. J Health Care Poor Underserved 2016; 27:53-61.

46. Komaromy M, Duhigg D, Metcalf A, et al.: Project ECHO (Extension for Community Healthcare Outcomes): a new model for educating primary care providers about treatment of substance use disorders. Subst Abuse 2016;37:20-24.

47. Socolovsky C, Masi C, Hamlish T, et al.: Evaluating the role of key learning theories in ECHO: a telehealth educational program for primary care providers. Prog Community Health Partnersh 2013;7:361-368.

Address correspondence to: Patrick J. O'Connor, MD, MA, MPH HealthPartners Institute 8170 33rd Avenue So., MS23301A Minneapolis, MN 55425

E-mail: patrick.j.oconnor@healthpartners.com 